\title{
Changes in the Network Eigen Entropy of resting-state functional brains across the lifespan
}

\author{
Yiming Fan, Hui Shen, Ling-li Zeng, Jian Qin,Zhiguo Luo,Dewen,Hu \\ College of Mechatronics and Automation, National University of Defense Technology, \\ Changsha, Hunan 410073, China.
}

fanyimingendeavor@126.com

Keywords: network eigen entropy (NEE), brain networks, fMRI, eigenvector centrality, graph theory.

\begin{abstract}
The brain is a dynamic and complex network formed by hierarchical interconnections among functional regions and neural systems across lifespan. Recent studies using graph theory explore the functional organization of resting-state brains have coherently indicated many non-random topological properties such as modular structure, central communication hubs, small-world property, scale-free network, and efficient network architecture. However these studies have focused mainly on partial brain attributes rather than integrated communication across brain regions and the organization or order of the brain. In this study, we explored the entire lifespan trajectory of human whole-brain organization used resting-state functional magnetic resonance imaging (rs-fMRI) and entropy analyses of graphic network. We found that the network eigen entropy (NEE) decreased from child to early middle aged but remained stable from middle aged to early old. Further, the slightly increased NEE was observed in the older people. Our findings not only revealed the U-shaped development and aging trajectory of functional organization in resting-state human that underpin variance in cognitive functions of brains across lifespan.
\end{abstract}

\section{Introduction}

The brain is inherently a dynamic and complex system across the lifespan, which can be conceptualized as a network of anatomically linked regions, in which the traffic between regions, during even at rest, creates and reshapes continuously complex functional networks of correlated dynamics. Complex network analysis has its origins in the mathematical study of networks known as graph theory. Therefore it is amenable to analysis brain network employing graph-based methods. We define brain regions and functional connections as nodes and links in a graph, respectively.

Recent studies using graph theory to explore the organization of resting-state functional brain networks have coherently indicated many non-random topological properties such as modular structure, central communication hubs, small-world property, scale-free network, efficient network architecture[1]. Meanwhile, resting-state functional magnetic resonance imaging (rs-fMRI) studies have researched age-related changes in functional connectivity patterns in the whole-brain network across the lifespan[2, 3]. However these studies have focused mainly on partial brain attributes rather than integrated communication across brain regions and the organization or order of the brain across the lifespan.

In this study, we explored the entire lifespan trajectory of the human whole-brain organization used rs-fMRI and entropy analyses of graphic network. As a significant demographic property, entropy in its purest information theoretical sense is a dimensionless quantity used for measuring uncertainty or ignorance about the state of a system. By implication, entropy is greater the more random a system is. Thus network entropy is a powerful explanatory tool for functional brain network since it provides a quantitative index of a dynamic system's randomness or disorder while simultaneously describing its informational characteristics .

\section{Materials and Methods}

MRI data acquisition. An available online public resting-state fMRI data sets were selected in the study, which were from the NKI / Rockland Sample (NKI-RS) ( http : //fcon.1000. projects. 
nitrc.org/ indi/ pro / nki. html). All approvals and procedures for collection and sharing of data were approved by the NKI institutional review board.

Data sets consisted of a total of 173 healthy subjects (age 7-85 years; mean age, 36.53 \pm 20.28 years; 102 males) (Table1). MRI data were acquired on a 3.0 T SIMENS Trio scanner. Structural images were acquired using a magnetization-prepared rapid gradient echo (MPRAGE) sequence (repetition time $[\mathrm{TR}]=2500 \mathrm{~ms}$, echo time $[\mathrm{TE}]=3.5 \mathrm{~ms}$, flip angle $=8^{\circ}$, thickness $=1.0 \mathrm{~mm}$, slices $=$ 192, matrix $=256 \times 256$, and FOV $=256 \mathrm{~mm}$ ). Functional images were acquired using echo-planar imaging $(\mathrm{EPI})$ sequence $(\mathrm{TR} / \mathrm{TE}=2500 / 30 \mathrm{~ms}, \mathrm{FA}=80, \mathrm{FOV}=216 \mathrm{~mm}$, matrix $=64 \times 64$, slices $=$ 38, thickness $=3.0 \mathrm{~mm})$. A 10-min $(260 \mathrm{TRs})$ resting state fMRI scan were collected for each subject. Subjects were instructed to relax and fixate on a crosshair while remaining as still as possible.

Table1 characteristics of subjects in the study

\begin{tabular}{cccc}
\hline Age group & Age range & Number of subjects & Gender(M/F) \\
\hline I & $7-17$ & 30 & $17 / 13$ \\
II & $18-28$ & 51 & $31 / 20$ \\
III & $30-45$ & 37 & $25 / 12$ \\
IV & $45-60$ & 24 & $15 / 9$ \\
V & $60-85$ & 30 & $14 / 16$ \\
\hline
\end{tabular}

Before data preprocessed, the first five functional images of each scan were discarded to remove possible $\mathrm{T} 1$ stabilization effects. Then, imaging data were preprocessed using SPM8 software (http://www.fil.ion.ucl.ac.uk/spm). Preprocessing steps included the removal of sudden spikes caused by large head motions, slicing time correction, motion correction, normalization, spatial smoothing(FWHM=6mm kernel). To reduce hardware noise, draining vessel effect, and motion artifacts in each gray matter voxel, We further regressed the following nuisance variables: 6-parameter motion, the white matter (WM), cerebrospinal fluid (CSF), and whole-brain signals [4].

Network Analyses. Given that the spatial resolution chosen to define network nodes (e.g. single voxel nodes, nodes defined by functional regions of interests (ROIs), or nodes based upon anatomical landmarks) could strongly influence the results[5], we compared network metrics across different node definitions. In the study, network "nodes" were defined at 2 different approaches: 1) a functional ROI approach consisting of 160 10-mm diameter functional brain regions defined based on a prior meta-analysis of fMRI activation studies[6]. This approach parcellated the brain into segregated functionally defined ROIs that covered most of the cortex, 2) a spatially constrained spectral clustering approach for group clustering whole brain resting-state fMRI data into functionally and spatially coherent regions[7]. This method incorporated a spatial constraint similar to those previously proposed for other ROI generation clustering methods [8] into the normalized cut (NCUT) method previously proposed for unconstrained clustering of resting-state.We constructed network matrices by calculating correlation matrices of resting-state BOLD signal fluctuations among network nodes. To remove spurious correlations, we set the matrix elements (correlations) with $\mathrm{p}$-values higher than a statistical threshold ( $p<0.05$, Bonferroni-corrected) to 1[9], otherwise we set the matrix elements to zero. Then a set of symmetric, undirected adjacent matrices representing the functional brain network were acquired. Due to the ambiguous biological explanation of negative correlations[10], we restricted our analysis to positive correlations.

Graph-theoretic centrality measures estimate the functional significance of a node to a network, where the simplest centrality measure is degree centrality. Degree centrality can be calculated by summing the number of edges connected to each node. However, the eigenvector centrality (EC) is more sophisticated than degree. Where degree gives a simple count of the number of connections a node has, EC acknowledges that not all connections are equal. In general, connections to people who are themselves influential will lend a person more influence than connections to less influential people. If we denote the centrality of no $i$ by $e_{i}$ then we can allow for this effect by making $e_{i}$ proportional to the average of the centralities of $i$ 's network neighbors. This metric uses 
the full information of unthresholded weighted networks and is defined by the eigenvector of the largest eigenvalue of a symmetric matrix

$$
e_{i}=\frac{1}{\lambda} \sum_{j \in N} a_{i j} e_{j}
$$

Where $\lambda$ is the largest eigenvalue (using the Perron-Frobenius theorem), $e$ is the corresponding eigenvector, and $a_{i j}$ is the element of adjacent matrix. In this study, NEE was used to measure the homogeneity of resting-state functional brain network. A resting-state functional brain NEE is defined as

$$
E=-\sum_{i=1}^{N} I_{i} \ln \left(I_{i}\right)
$$

Where $I_{i}$ is the strength of node $i$, defined as

$$
I_{i}=e_{i} \div \sum_{i=1}^{N} e_{i}
$$

First, we normalized NEE to remove the effects of the number of nodes. Obviously, maximum NEE was got, when the network completely average,

$$
\text { that is } I_{i}=\frac{1}{N}, E_{\max }=-\sum_{i=1}^{N} \frac{1}{N} \ln \frac{1}{N}=\ln N
$$

Theoretically, when no link exists in the network, minimum $N E E$ was got, $E_{\min }=0$.

$$
\text { Then, } \bar{E}=\frac{E-E_{\min }}{E_{\max }-E_{\min }}=\frac{E}{E_{\max }}
$$

Where $\bar{E}$ is the normalized NEE (NNEE).

Brain networks with higher NEE are thought to more disorder, and oppositely, brain networks with lower $N E E$ are thought to more well-organized. So $N E E$ is a powerful explanatory tool for exploring the lifespan developmental dynamics of brain networks.

It is crucial to note that values of $N E E$ are greatly influenced by basic network characteristics, such as the number of nodes and links, and the degree centrality distribution. The significance of network statistics should be established by comparison with statistics calculated on null-hypothesis networks. In the study, we used random networks as the null-hypothesis. Random networks we used share the size, density and binary degree centrality distribution of the resting-state functional brain network[11]. Note, however, that this network may have a different eigenvector centrality distribution. Brain networks, especially for the adults, demonstrate significantly different NEE from random networks (Fig 1).

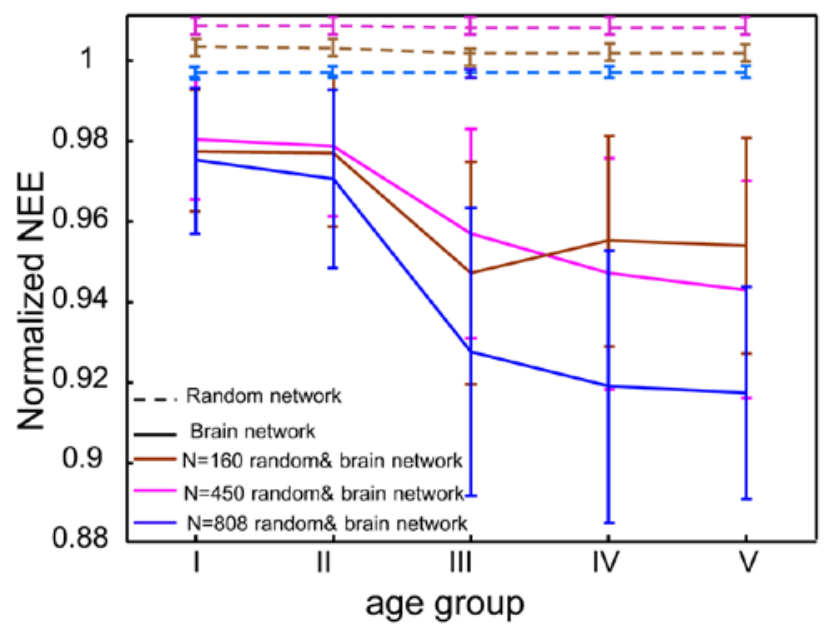

Fig.1 Normalized network eigen entropy (NNEE) achieved for five different age ranges (I (7-17), II (18-28), III (30-45) IV (45-60) V (60-85)) at three different spatial scales network (the number of 
ROI $N$ equal 160 - brownness, $N=500$-red, $N=808$-blue). The changes of five age ranges for random work are shown with dashed lines. The network properties of the number of node, degree centrality are the same as the corresponding brain network. The changes of five age ranges for resting-state brain network are shown with solid lines. We normalized each individual NEE, as it was affected by the number of node.

Statistical analyses. To determine the changes of the NEE across the lifespan precisely, a general linear model (GLM) was used for each metric as follows. To explore linear and quadratic age effects, we used multiple linear regressions that modeled the targeted property with age and age ${ }^{2}$ as predictors along with three other nuisances variables that included sex (male 1 , female 0 ), head-motion, mean frame-wise displacement (meanFD) [12], and temporal signal-to-noise ratio (tSNR) [13], which was used to estimate data quality and mitigate the effects of nuisance variables. Notably, the meanFD of all subjects was $<0.50 \mathrm{~mm}$, which indicates that the subjects included in the study had rather small head motions. Specifically, the GLM models were separately formulated as follows:

$$
\begin{gathered}
E C=\beta_{0}+\beta_{1} \times a g e+\beta_{2} \times \operatorname{sex}+\beta_{3} \times \text { meanFD }+\beta_{4} \times t S N R \\
E C=\beta_{0}+\beta_{1} \times a g e+\beta_{2} \times a g e^{2}+\beta_{3} \times \operatorname{sex}+\beta_{4} \times \text { meanFD }+\beta_{5} \times t S N R
\end{gathered}
$$

For each eigenvector centrality, $\mathrm{T}$ statistic for coefficients of age in equation (6) was used to measure the significance of linear developmental changes.T statistic for coefficients of age ${ }^{2}$ in equation (7) also was used to measure the significance of quadratic developmental changes when the linear effects of age had been removed. The brain regions showing significant age-related changes in the EC were identified across the lifespan (see Fig 2, $p<0.05$,FDR-corrected).
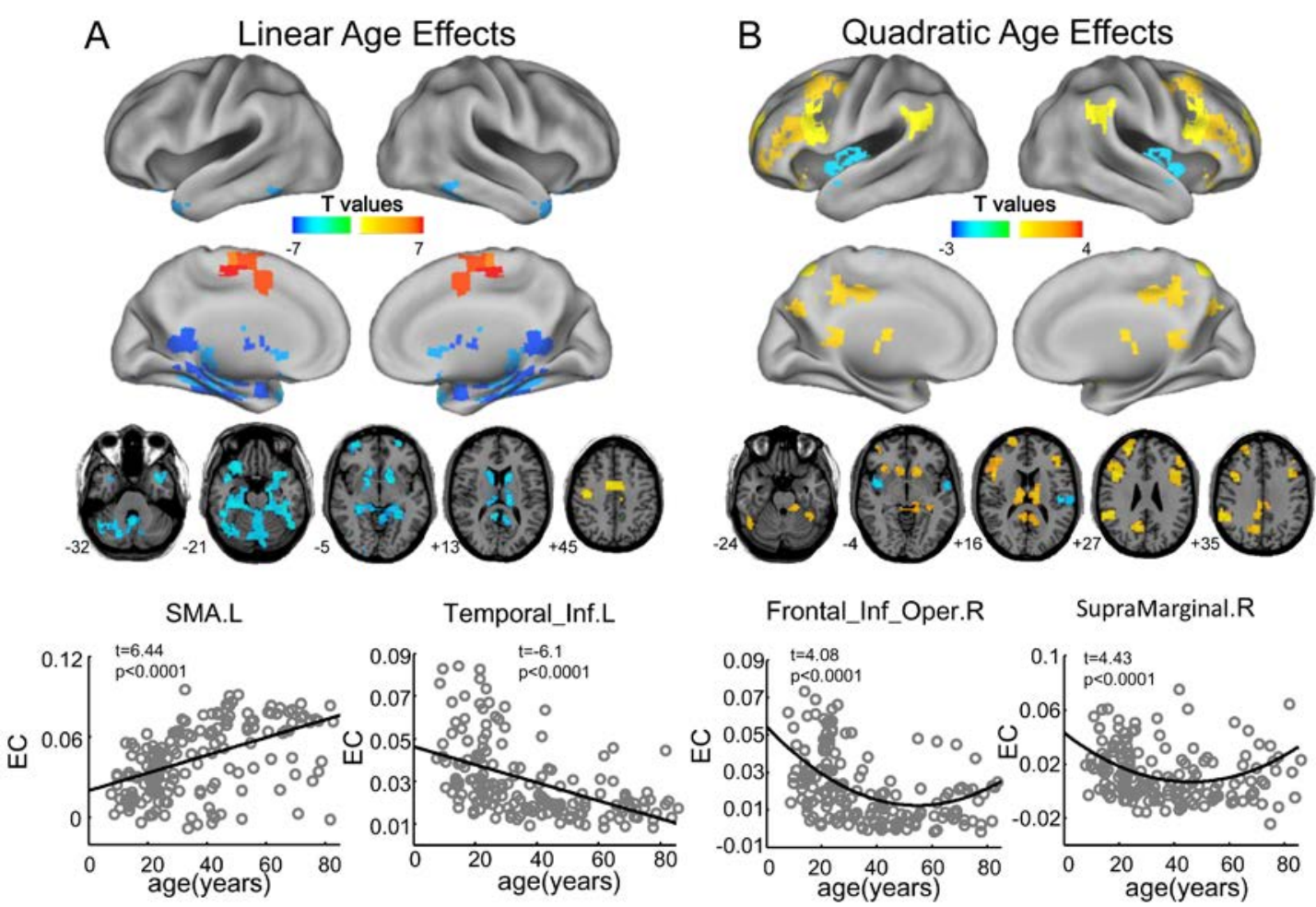

Fig.2 EC changes across the lifespan at the spatial scale of node number 808. (A) The regions display significant linear age effects. (B) The regions display significant quadratic effects. $p<0.05$, FDR correction was finished to correct for multiple comparisons. The bottom row reveals the dynamic development changes of certain, typical regions. SMA.L, left Sup_Motor_Area. The curve fits are revealed by the dark lines. The gray dots represent the values of EC for each subject after regressing out meanFD, sex, tSNR. The color bars represent the changes of t-values (T statistic).

Model selection curve fitting and graphing. Model selection for NEE curves utilized Akaike information criterion( $A I C)$, which allows comparison of non-nested models. The AIC $(2 k-2 \ln (L)$, where $k=$ number of parameters and $L=$ maximum like hood of model) provides a relative goodness 
of fit estimation for different models when applied to the same data. It takes into account both descriptive accuracy and parsimony, since it carries a penalty for increasing the number of free parameters. The absolute $A I C$ values are not particularly meaningful since they are specific to the data set being modeled. The relative $A I C$ values $\left(\triangle A I C_{i}=A I C_{i}-\min A I C\right.$, however, can be used to rank models. The relatively best model is assigned a $\triangle A I C_{i}$ of zero. The $\triangle A I C_{i}$ can be interpreted as the probability that a model is the best model among the set of examined models. Using these models, four plausible models with either two or three, four parameters were analyzed (Table2).

All curve fitting, graphing and statistical testing was carried out using MATLAB 10.0 (R2010a) and the MATLAB Statistics and Curve Fitting toolboxes and in-house MATLAB code.

\section{Results}

The curve fits of Normalized NEE utilized four different models and were shown on three different spatial scales (160 nodes, 500 nodes, and 808 nodes). The results are shown in Fig.3. Model selection analyses were carried out by using AIC . These analyses showed that NEE across lifespan are best fit by quadratic model (Table 2).

The system of brain was random from child to early adulthood, perhaps in this stage, we were learning all kinds of skills and beginning to distinguish.The organization of the human brain was more and more orderly and kept stable in a period time of middle aged to early old.The result from older revealed the functional connectivity related to cognitive function lost possibly. So the organization of brain showed disorder again.
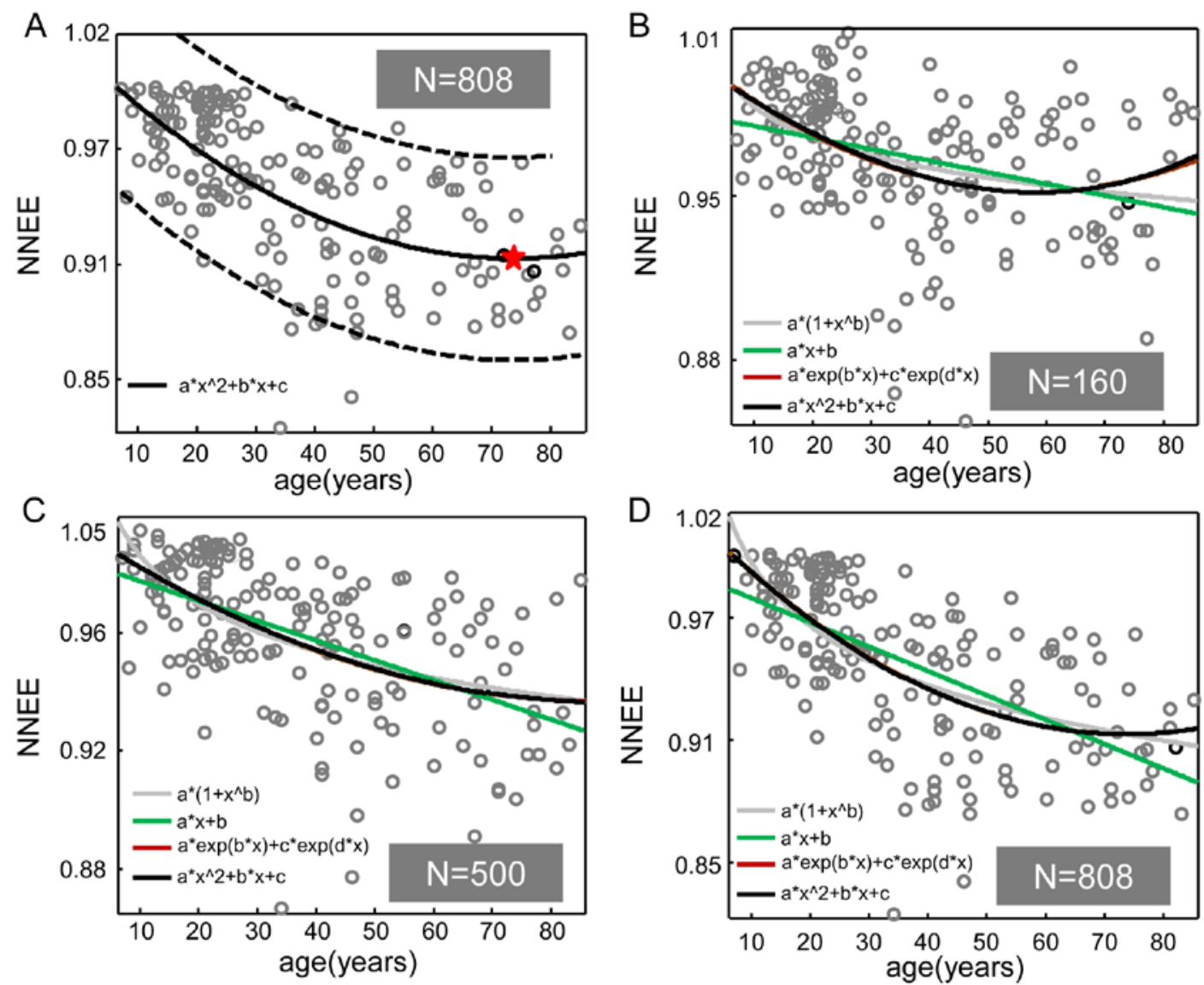

Fig.3 Individual network eigen entropy (NEE) levels between the ages of 7 to 85 years. Chronological age is shown on the $x$-axis and the Normalized NEE on the $y$-axis. The gray circles represent the adjusted results of each subject after regressing out head motion, sex and temporal signal-to-noise ratio. (A) Normalized NEE curve at the spatial scale of 808 nodes. The fit for the quadratic equation ( $a x^{2}+b x+c ; R^{2}=0.42$, permutation test, $p<0.001, \Delta \mathrm{AIC}=0.00$ ) is shown with a 
solid black line. The red star is located the inflection point of age. The 95\% prediction limits are shown with dashed lines. (B)-(D) normalized NEE curves at the three different spatial scales. (B: the number of nodes $N-160, \mathrm{C}: N-500$ and D: $N-808)$ Fitted curves for functions included in model selection are shown (Power-light gray; linear-green; Quadratic-black; double Exponential-brown)

Table2. Model selection and curve fitting

\begin{tabular}{|c|c|c|c|c|c|c|c|c|c|c|}
\hline \multirow[b]{2}{*}{ ROI number } & \multirow[b]{2}{*}{ Fit name } & \multirow[b]{2}{*}{ Equation } & \multirow[b]{2}{*}{ SSE } & \multirow[b]{2}{*}{$R^{2}$} & \multirow[b]{2}{*}{$\operatorname{Adj} \cdot R^{2}$} & \multirow[b]{2}{*}{$\Delta \mathrm{AIC}$} & \multicolumn{4}{|c|}{ Parameter } \\
\hline & & & & & & & a & & c & d \\
\hline \multirow{4}{*}{160} & $a^{*}\left(1+x^{\wedge} b\right)$ & Power & 0.0025 & 0.14 & 0.14 & 3.79 & 0.081 & -0.037 & - & - \\
\hline & $a * x+b$ & Linear & 0.0023 & 0.12 & 0.11 & 8.76 & $-7.06 e-5$ & 0.155 & - & - \\
\hline & $a^{*} x^{\wedge} 2+b^{*} x+c$ & Quadratic & 0.0024 & 0.17 & 0.16 & 0.00 & $2.41 \mathrm{e}-6$ & $-2.79 \mathrm{e}-3$ & 0.158 & - \\
\hline & $a^{*} \exp \left(b^{*} x\right)+c^{*} \exp \left(d^{*} x\right)$ & Exponential & 0.0024 & 0.17 & 0.16 & 1.76 & 0.145 & -0.003 & 0.013 & 0.013 \\
\hline \multirow{4}{*}{500} & $a^{*}\left(1+x^{\wedge} b\right)$ & Power & 0.00079 & 0.29 & 0.28 & 1.89 & 0.049 & -0.056 & - & - \\
\hline & $a^{*} x+b$ & Linear & 0.00079 & 0.30 & 0.28 & 1.35 & $-6.7 e-6$ & 0.0924 & - & - \\
\hline & $a^{*} x^{\wedge} 2+b^{*} x+c$ & Quadratic & 0.00077 & 0.28 & 0.30 & 0.00 & $7.53 \mathrm{ee}-7$ & $-1.3 e-3$ & 0.093 & - \\
\hline & $a^{*} \exp \left(b^{*} x\right)+c^{*} \exp \left(d^{*} x\right)$ & Exponential & 0.00077 & 0.30 & 0.30 & 2.05 & 0.057 & -0.004 & 0.037 & 0.003 \\
\hline \multirow{4}{*}{808} & $a^{*}\left(1+x^{\wedge} b\right)$ & Power & 0.00071 & 0.40 & 0.39 & 3.77 & 0.039 & -0.10 & - & - \\
\hline & $a * x+b$ & Linear & 0.00073 & 0.38 & 0.37 & 9.29 & $-7.96 e-5$ & 0.069 & - & - \\
\hline & $a^{*} x^{\wedge} 2+b^{*} x+c$ & Quadratic & 0.00068 & 0.42 & 0.41 & 0.00 & $1.31 \mathrm{e}-6$ & $-1.9 \mathrm{e}-3$ & 0.071 & - \\
\hline & $a^{*} \exp \left(b^{*} x\right)+c^{*} \exp \left(d^{*} x\right)$ & Exponential & 0.00068 & 0.42 & 0.41 & 2.03 & 0.053 & $-5.98 \mathrm{e}-3$ & 0.018 & 0.007 \\
\hline
\end{tabular}

* $\mathrm{SSE}$ - the error sum of square. Adj. $R^{2}$-adjusted $R^{2}$

\section{Conclusion}

In the study, Our findings suggest NEE is a quantitative index to represent a dynamic system's randomness or disorder. The NEE is a effective index to measure the developmental order of the human brain.

\section{References}

[1] van den Heuvel, M.P. and O. Sporns, An anatomical substrate for integration among functional networks in human cortex. The Journal of neuroscience, 2013. 33(36): p. 14489-14500.

[2] Zuo, X.-N., et al., Growing together and growing apart: regional and sex differences in the lifespan developmental trajectories of functional homotopy. The Journal of neuroscience, 2010. 30(45): p. 15034-15043.

[3] Wang, L., et al., Decoding lifespan changes of the human brain using resting-state functional connectivity MRI. PloS one, 2012. 7(8): p. e44530-e44530.

[4] Zeng, L.-L., et al., Identifying major depression using whole-brain functional connectivity: a multivariate pattern analysis. Brain, 2012. 135(5): p. 1498-1507.

[5] Smith, S.M., et al., Network modelling methods for FMRI. Neuroimage, 2011. 54(2): p. 875-891.

[6] Dosenbach, N.U., et al., Prediction of individual brain maturity using fMRI. Science, 2010. 329(5997): p. 1358-1361.

[7] Craddock, R.C., et al., A whole brain fMRI atlas generated via spatially constrained spectral clustering. Human brain mapping, 2012. 33(8): p. 1914-1928.

[8] Tomassini, V., et al., Diffusion-weighted imaging tractography-based parcellation of the human lateral premotor cortex identifies dorsal and ventral subregions with anatomical and functional specializations. The Journal of neuroscience, 2007. 27(38): p. 10259-10269.

[9] Cao, M., et al., Topological organization of the human brain functional connectome across the lifespan. Developmental cognitive neuroscience, 2014. 7: p. 76-93.

[10] Murphy, K., et al., The impact of global signal regression on resting state correlations: are anti-correlated networks introduced? Neuroimage, 2009. 44(3): p. 893-905.

[11] Maslov, S. and K. Sneppen, Specificity and stability in topology of protein networks. Science, 2002. 296(5569): p. 910-913.

[12] Power, J.D., et al., Spurious but systematic correlations in functional connectivity MRI 
networks arise from subject motion. Neuroimage, 2012. 59(3): p. 2142-2154.

[13] Van Dijk, K.R., M.R. Sabuncu, and R.L. Buckner, The influence of head motion on intrinsic functional connectivity MRI. Neuroimage, 2012. 59(1): p. 431-438. 